\title{
Optimal Dividend and Financing Control Problem in The Risk Model with Non-Cheap Proportional Reinsurance
}

\author{
Ye Liu' ${ }^{1}$, Danfeng Zhao ${ }^{2}$ \\ ${ }^{1}$ Hebei University of Technology, School of Sciences, Beichen district, Tianjin 300401, China \\ ${ }^{2}$ School of Sciences, Hebei University of Technology, Beichen district, Tianjin 300401, China
}

\begin{abstract}
In this paper, we consider the optimal dividend and financing control problem in the risk model with non-cheap proportional reinsurance and two kinds of transaction costs. The company control its reserves by paying dividends, issuing equity and taking reinsurance. In our model, the objective is to find the strategy which maximizes the expected present value of the dividends payout minus the equity issuance until the time of ruin. We solve the optimal control problem and identify the optimal strategy by constructing two categories of suboptimal control problems, one is the classical model without equity issuance,the other never goes bankrupt by equity issuance.
\end{abstract}

Keywords: dividend, equity issuance, non-cheap proportional reinsurance, HJB equation, optimal strategy

\section{Introduction}

Optimal dividend strategy, as one major public concern to assess the stability of companies that take on risks, has become an increasingly popular topic in actuarial research. Its origin can be traced as early as the work of De Finetti [1], which introduced a discrete-time model for optimal dividend. De Finetti showed that the optimal strategy is a barrier strategy and determined the optimal level of the barrier by maximizing the expected discounted dividends paid to shareholders. Since then the optimal dividend strategy has been studied extensively. Some recent works include Højgaard and Taksar [2], Gerber and Shiu [3], Cadenillas et al.[4], Avanzi et al.[5] and so on.

Meanwhile, in the real financial market, equity issuance is an important approach for the insurance company to earn profit and reduce risk. Both equity issuance and dividend are important issues in modeling insurance risk. Sethi and Taksar [6] recently considered the model for the company that controls its risk exposure by issuing new equity and paying dividends to shareholders and discussed the problem using singular and impulse controls. Løkka and Zervos [7] studied the combined optimal dividend and equity issuance problem by taking into account the possibility of bankruptcy. The aforementioned authors mainly focused on developing optimal dividend and equity issuance strategies. They did not consider reinsurance.

Reinsurance plays a significant role in both theory and practice of insurance risk modeling. It is a means by which a direct insurance company can transfer the risks from their liabilities to a second insurance carrier. The three popular types of reinsurance strategies are stop-loss reinsurance, proportional reinsurance and excess-of-loss reinsurance. The academia and practitioners have paid more attention to the proportional reinsurance and excess-of-loss reinsurance. Some works on the excess-of-loss reinsurance are Asmussen et al.[8], Choulli et al.[9], Centeno [10] and so on. Some literature on the proportional reinsurance includes He and Liang [11], [12] and so on. He and Liang [11], [12]incorporated the proportional reinsurance strategy in the combined dividend and equity issuance problem using both singular and mixed singular-impulse controls.

Motivated by these works, we consider the optimal dividend and financing control problem of an insurance company. We assume that the company can control its reserves by paying dividends, issuing equity and taking non-cheap proportional reinsurance. Moreover, there exists a minimal reserve requirement. And some costs will be incurred: reinsurance company will need more premium for the risk ceded by the insurer; fixed cost is generated by advisory and consulting fees when payingdividends; proportional transaction costs are generated by the tax. In this paper, we consider the dividends payout and the equity issuance as the reflecting and absorbing boundaries of the reserve process, respectively. Firstly, we study the solutions of two models: one is diffusion control model without equity issuance, the other stands for the model with equity issuance to meet the minimal reserve requirement, so it never goes bankrupt. Our objective is to maximize the expected present value of the dividends payout minus the equity issuance until the time of ruin. Then we prove that the value functions and the optimal strategies are the solutions of the two control problems. We provide a rigorous and detailed mathematical analysis for the combined effect of the optimal dividend, equity issuance and non-cheap proportional reinsurance strategies.

The rest of the paper is organized as follows. In Section 2, we introduce the control model of an insurance company with non-cheap proportional reinsurance. In Section 3, we present two lemmas for proving the main results of this paper. In Section 4, we construct solutions of two categories of suboptimal models. In Section 5, we verify the value function and the optimal strategy with the corresponding solution in 


\section{International Journal of Science and Research (IJSR) \\ ISSN (Online): 2319-7064}

Index Copernicus Value (2013): 6.14 | Impact Factor (2015): 6.391

either category of suboptimal models, tightly connecting with the relationships among the parameters.

\section{The Model}

Let $\left(\Omega, \mathrm{F},\left\{\mathrm{F}_{t}\right\}_{t \geq 0}, P\right)$ be a filtered probability space and $B_{t}$ is a standard Brownian motion on this probability space, where $\mathrm{F}_{t}$ represents the information available at time $t$ and any decision made up to time $t$ is based on this information. To pay dividends to the share holders, the insurance company has to determine the times and amounts of dividend payments. A dividend stream is defined by $L:=\left\{\left(\tau_{\mathrm{i}}, \xi_{\mathrm{i}}\right) \mathrm{i}=1,2, \ldots \ldots\right\}$, where $\tau_{\mathrm{i}}, \xi_{\mathrm{i}}$ are the time and amount of the $i$ th dividend payment, respectively. We assume that $\left\{\tau_{i}, \mid i=1,2, \ldots ..\right\}$ is a sequence of increasing stopping times and $\left\{\xi_{i}, \mid i=1,2, \ldots ..\right\}$ is a sequence of non-negative,i.i.d random variables.Let $L_{t}$ denote the total amount of dividends paid until time $t$. Then we can define $L_{t}:=\sum_{i=1}^{\infty} I_{\left\{\tau_{\mathrm{i}} \leq t\right.} \xi_{\mathrm{i}}$, where $I_{E}$ is the indicator function of the event $E$. We suppose the liquid reserves of the insurance company must satisfy some minimal reserve requirement. In this case, we assume that the company needs to keep its reserves above $m, m>0$ is the minimal reserve requirement. The company is considered bankrupt as soon as the reserves fall below $m$. However, to avoid bankruptcy, the company should issue some equity. We denote $G_{t}$ as the total amount raised by issuing equity from time 0 to $t$. We assume that the process $\left\{G_{t}, t \geq 0\right\}$ is $\left\{\mathrm{F}_{t}\right\}_{t \geq 0}$-adapted, increasing, right-continuous with left limits and $G(0)=0$. By the reinsurance strategy $a_{t} \in[0,1]$ (the proportional retention level), the amount of dividend $L_{t}$ and equity issuance $G_{t}$, the liquid reserve of the insurance company evolves according to the stochastic equation,

$$
R_{t}=x+\int_{0}^{t}\left[a_{s} \lambda-(\lambda-\mu)\right] d_{s}+\int_{0}^{t} a_{s} d B_{s}-\sum_{n=1}^{\infty} I_{\left\{\tau_{i} \leq t\right\}} \xi_{\mathrm{i}}+G_{t}
$$

where $x$ is the initial reserve, $\mu>0$ is the relative safety loading of the insurer and $\lambda$ is the relative safety loading of the reinsurer.

The proportional reinsurance is cheap when $\lambda=\mu$. We consider the case of $\lambda>\mu$ which is called non-cheap proportional reinsurance.

We will define an admissible strategy as follows.

A strategy $\pi=\left\{a_{t}, t \geq 0 ; \tau_{1}, \tau_{2}, \ldots . . ; \xi_{1}, \xi_{2}, \ldots . . ; \mathrm{G}\right\}$ is said to be admissible if

(i) $\left\{a_{t}\right\}$ is an $\left\{\mathrm{F}_{t}\right\}_{t \geq 0}$-adapted process and $P\left(a_{t} \in[0,1]\right)=1$ for any $t \geq 0$.

(ii)For each $\mathrm{i}=1,2, \ldots . .,\left\{\tau_{\mathrm{i}} \leq t\right\} \in F_{t}$ and $\xi_{\mathrm{i}} \in \mathrm{F}_{\tau_{\mathrm{i}}}$. (iii) $\left\{L_{t}\right\}$ is $\left\{\mathrm{F}_{t}\right\}_{t \geq 0}$ - progressively measurable, increasing and cádlág, $\left\{G_{t}, t \geq 0\right\}$ is $\left\{\mathrm{F}_{t}\right\}_{t \geq 0}$-adapted, increasing, cádlág and $\xi_{\mathrm{i}} \leq R_{\tau_{i}^{-}}-m$.

(iv) $\mathrm{P}\left(\lim \tau_{\mathrm{i}} \leq t\right)=0, \forall t \geq 0$

(v) $\square L_{t} \cdot \square G_{t}=0$, here $\square L_{t}=L_{t}-L_{t^{-}}, \square G_{t}=G_{t}-G_{t^{-}}$.

We write $\Pi(x)$ for the space of these admissible policies. For each $\pi \in \Pi(x)$, we write $R^{\pi}:=\left\{R_{t}^{\pi} \mid \mathrm{t} \geq 0\right\}$ for the surplus progress of the company associated with $\pi$. The surplus progress is written as follows,

$R_{t}^{\pi}=x+\int_{0}^{t}\left[a_{s}^{\pi} \lambda-(\lambda-\mu)\right] d_{s}+\int_{0}^{t} a_{s}^{\pi} d B_{s}-\sum_{n=1}^{\infty} I_{\left\{\tau_{i}^{\pi} \leq t\right\}} \xi_{\mathrm{i}}^{\pi}+G_{t}^{\pi}$.

The ruin time correspond to $\pi$ is defined as: $\tau^{\pi}:=\inf \left\{t \geq 0: R_{t}^{\pi}<m\right\}$ and $\tau^{\pi}$ is an $\mathrm{F}_{t}-$ stopping time. For each dividend payment, we have to pay a fixed set-up cost $K \in(0, \infty)$, which is independent of the amount of the payment. Let $\beta_{1}<1$ be a positive number. Then $1-\beta_{1}$ is the tax rate if the dividend is taxed. Consequently, the amount of the money that the shareholder receives is $-K+\beta_{1} \xi_{i}^{\pi}$ if the amount $\xi_{i}^{\pi}$ of the liquid assets is distribute. In the meanwhile, the shareholders must pay out $\beta_{2} g\left(\beta_{2}>1\right)$ to meet the amount of $g$ as new equity of the company. $\beta_{2}$ is the proportional transaction cost generated by the issuance of equity.

So our optimal control problem is to maximize the expected present value of the dividends payout minus the equity issuance before bankruptcy, i.e. we need to find $\pi \in \Pi$ maximizing the following performance function as

$V(x, \pi)=E\left[\sum_{i=1}^{\infty} e^{-\delta \tau_{i}^{\pi}}\left(-K+\beta_{1} \xi_{i}^{\pi}\right) I_{\left\{\tau_{i}^{\pi} \leq \tau^{\pi}\right\}}-\int_{0}^{\tau^{\pi}} \mathrm{e}^{-\delta \mathrm{s}} \beta_{2} d G_{s}^{\pi}\right]$.

The optimal value function is defined as

$$
V(x)=\sup _{\pi \in \Pi} V(x, \pi) \text {. }
$$

In addition, the minimal reserve requirement asks for $V(x)=0$, for $\forall x<m$. To solve the optimization problem, our must determine the value function $V(x)$ and the optimal strategy $\pi^{*}$ satisfies $V(x)=V\left(x, \pi^{*}\right)$.

Next, we will divide $\lambda>\mu$ into two parts: $\mu<\lambda<2 \mu$ and $\lambda \geq 2 \mu$.

(I) We discuss the case of $\mu<\lambda<2 \mu$. It includes two situations: (i) $x_{1}>d>x_{0}$ and (ii) $0 \leq d<x_{0}$, where

$$
x_{0}=G\left(\frac{2 \mu-\lambda}{2 \delta}\right), G(u)=\int_{0}^{u} \frac{\delta \mathrm{y}+\lambda-\mu}{\left(\delta+\frac{\lambda^{2}}{2 \sigma^{2}}\right) \mathrm{y}+\lambda-\mu} d y, u \geq 0,
$$

$d$ is a nonnegative constant.

First, we consider the situation (i):

\section{Volume 5 Issue 6, June 2016 www.ijsr.net}




\section{International Journal of Science and Research (IJSR) \\ ISSN (Online): 2319-7064}

Index Copernicus Value (2013): 6.14 | Impact Factor (2015): 6.391

((i) will be discussed in Section 3 and Theorem 4.1 and Theorem 4.2. (ii) and (II): $\lambda \geq 2 \mu$ will be discussed after that.)

\section{Two Primary Lemmas}

In this section we give two main lemmas before proving the Theorems in Section 4.

\section{Lemma 3.1.}

There exists a unique $x_{1_{*}}=x_{1_{*}}\left(\delta, \mu, \sigma, \beta_{1}, K\right)>m$ satisfying the following equation in $x_{1}$,

$\frac{\beta_{1} b_{2}}{b_{1}\left(b_{2}-b_{1}\right)} e^{b_{1}\left(m-x_{1}\right)}+\frac{\beta_{1} b_{1}}{b_{2}\left(b_{1}-b_{2}\right)} e^{b_{2}\left(m-x_{1}\right)}=0$,

where $b_{1}=\frac{-\mu-\sqrt{\mu^{2}+2 \delta \sigma^{2}}}{\sigma^{2}}, \quad b_{2}=\frac{-\mu+\sqrt{\mu^{2}+2 \delta \sigma^{2}}}{\sigma^{2}}$.

Proof. Denote the left-hand side of (3.1)by $k\left(x_{1}\right)$.

Differentiating $k\left(x_{1}\right)$ with respect $\mathrm{t} x_{1}$, we have

$k^{\prime}\left(x_{1}\right)=\frac{-\beta_{1} b_{2}}{b_{2}-b_{1}} e^{b_{1}\left(m-x_{1}\right)}+\frac{\beta_{1} b_{1}}{b_{2}-b_{1}} e^{b_{2}\left(m-x_{1}\right)}<0$.

Then $k\left(x_{1}\right)$ is a strictly decreasing function of $x_{1} \cdot k\left(x_{1}\right)$

reaches its maximum at $m$ on $[m,+\infty)$. We deduce

$k\left(x_{1}\right) \rightarrow-\infty$, as $x_{1} \rightarrow \infty$ and

$k(m)=\frac{\beta_{1} b_{2}}{b_{1}\left(b_{2}-b_{1}\right)}+\frac{\beta_{1} b_{1}}{b_{2}\left(b_{1}-b_{2}\right)}>0 ，$

thus (3.1) has a unique solution $x_{1_{*}}$ and $x_{1_{*}}>m$.

\section{Lemma 3.2}

There exists a unique $x_{1_{* *}}=x_{1_{* *}}\left(\delta, \mu, \sigma, \beta_{1}, \beta_{2}\right)>m$ satisfying the following equation in $x_{1}$,

$\frac{\beta_{1} b_{2}}{b_{2}-b_{1}} e^{b_{1}\left(m-x_{1}\right)}+\frac{\beta_{1} b_{1}}{b_{1}-b_{2}} e^{b_{2}\left(m-x_{1}\right)}=\beta_{2}$,

where $b_{1}, b_{2}$ are the same as in Lemma 3.1.

Proof. Denote the left-hand side of (3.2) by $h\left(x_{1}\right)$.

Differentiating $h\left(x_{1}\right)$ with respect to $x_{1}$, we have $h^{\prime}\left(x_{1}\right)=\frac{-\beta_{1} b_{2} b_{1}}{b_{2}-b_{1}} e^{b_{1}\left(m-x_{1}\right)}-\frac{\beta_{1} b_{1} b_{2}}{b_{1}-b_{2}} e^{b_{2}\left(m-x_{1}\right)}>0$.

Then $h\left(x_{1}\right)$ is a strictly increasing function of $x_{1} \cdot h\left(x_{1}\right)$ reaches its minimum at $m$ on $[m,+\infty)$. We deduce

$h\left(x_{1}\right) \rightarrow+\infty$, as $x_{1} \rightarrow \infty$, and

$h(m)=\frac{\beta_{1} b_{2}}{b_{2}-b_{1}}+\frac{\beta_{1} b_{1}}{b_{1}-b_{2}}=\beta_{1}<\beta_{2}$, thus (3.2) has a unique solution $x_{1_{* *}}$ and $x_{1_{* *}}>m$.

\section{Two categories of suboptimal solutions}

In this section, we consider two categories of suboptimal control problems.

Let $\pi_{A}=\left\{a_{A}, L_{A}, 0\right\} \in \Pi$ be the control process for the company in which equity issuance is not permitted.We define the associated optimal value function as
$V_{A}(x)=\sup _{\pi_{A} \in \Pi} V\left(x, \pi_{A}\right)$, for $x \geq m$.

Let $\pi_{B}=\left\{a_{B}, L_{B}, \mathrm{G}_{\mathrm{B}}\right\} \in \Pi$ be the control process for the company with equity issuance procedures. In this case, the insurance company will never go bankrupt. The associated optimal value function is $V_{B}(x)=\sup _{\pi_{B} \in \Pi} V\left(x, \pi_{B}\right), x \geq m$.

According to (2.4), it follows that $V(x) \geq \max \left\{V_{A}(x), V_{B}(x)\right\}$ for $x \geq m$.

The two suboptimal solutions will play a key role in constructing the optimal policy $\pi^{*}$. Thus we will first study the solutions to the two suboptimal control problems.

\subsection{The solution to the problem without equity issuance}

In this subsection, our objective is to maximize the expected discounted dividends payout.

Theorem 4.1. We assume $x_{1_{*}} \leq x_{1_{m+}}$, where

$x_{1_{*}}$ and $x_{1_{* *}}$ are defined in Lemmas 3.1. and 3.2. Then the function $f$ defined by

$$
f(x)=\left\{\begin{aligned}
f_{1}(x)= & C_{1}\left(x_{1_{*}}\right) e^{b_{1}\left(x+d-x_{0}\right)}+C_{2}\left(x_{1_{*}}\right) e^{b_{2}\left(x+d-x_{0}\right)}, \\
& m \leq x<x_{1_{*}}, \\
f_{2}(x)= & \beta_{1}\left(x-x_{1_{*}}\right)+f_{1}\left(x_{1_{*}}\right), \\
& x \geq x_{1_{*}},
\end{aligned}\right.
$$

satisfies the following HJB equation and the boundary conditions for $x \geq m$,

$$
\begin{aligned}
& \max \left\{\max _{a \in[0,1]}\left\{\frac{1}{2} \sigma^{2} a^{2} f^{\prime \prime}(x)+[a \lambda-(\lambda-\mu)] f^{\prime}(x)-\delta f(x)\right\}, \beta_{1}-f^{\prime}(x),\right. \\
& \mathfrak{I} f(x)-f(x)\}=0, \\
& f(m)=0 .
\end{aligned}
$$

Moreover, for $x \geq m$,

$f^{\prime}(x) \leq \beta_{2}$,

where $b_{1}, b_{2}$ are the same as in Lemma 3.1, $C_{1}\left(x_{1_{*}}\right), C_{2}\left(x_{1_{*}}\right)$ are defined by

$$
\begin{aligned}
& C_{1}\left(x_{1_{*}}\right)=\frac{\beta_{1} b_{2}}{\mathrm{e}^{b_{1}\left(x_{1_{*}}-x_{0}+d\right)} b_{1}\left(b_{2}-b_{1}\right)}, C_{2}\left(x_{1_{*}}\right)=\frac{\beta_{1} b_{1}}{\mathrm{e}^{b_{2}\left(x_{1 *}-x_{0}+d\right)} b_{2}\left(b_{1}-b_{2}\right)} \\
& \mathfrak{I} f(x)=\sup _{m \leq y \leq x}\left\{-K+\beta_{1}(x+y)+f(y)\right\} \text {. }
\end{aligned}
$$

Proof. By the standard theory of optimal control, we use the same method as in Wendell and Fleming [13] and Højgaard and Taksar [2] to get a function $f$ satisfying the following HJB equations,

$$
\begin{aligned}
& \max _{a \in[0,1]}\left\{\frac{1}{2} \sigma^{2} a^{2} f^{\prime \prime}(x)+[a \lambda-(\lambda-\mu)] f^{\prime}(x)-\delta f(x)\right\}=0, \\
& \quad m \leq x<x_{1}, \\
& f^{\prime}(x)=\beta_{1}, \quad x \geq x_{1}, \\
& f^{\prime \prime}(x)=0, \quad x \geq x_{1} .
\end{aligned}
$$

Then differentiating w.r.t $a$ for the first equation of (4.1.5), we can find $a(x)=\frac{-\lambda f^{\prime}(x)}{\sigma^{2} f^{\prime \prime}(x)}$.

Since $a(x)$ belongs to $[0,1]$, putting the expression $a(x)$ 


\section{International Journal of Science and Research (IJSR) \\ ISSN (Online): 2319-7064}

Index Copernicus Value (2013): 6.14 | Impact Factor (2015): 6.391

into the first equation of (4.1.5), we get

$-\frac{\lambda^{2} f^{\prime}(x)}{2 \sigma^{2} f^{\prime \prime}(x)}-c \frac{f(x)}{f^{\prime}(x)}+(\mu-\lambda)=0$

Denoting $p(x)=\frac{f(x)}{f^{\prime}(x)}$, we get that

$p^{\prime}(x)=\frac{\left(\mathrm{c}+\frac{\lambda^{2}}{2 \sigma^{2}}\right) p(x)+\lambda-\mu}{c p(x)+\lambda-\mu}, x \geq m$.

By a simple calculation, we find that there exists a nonnegative constant $d$ such that

$$
p(x)=\frac{f(x)}{f^{\prime}(x)}=G^{-1}(x+d), \quad x \geq m,
$$

where $G^{-1}(\cdot)$ denote the inverse function of $G$.

We have $a(x)=\frac{2}{\lambda}\left[c G^{-1}(x+d)+\lambda-\mu\right]$.

Since $a(x) \in[0,1]$, we have $x+d \leq x_{0}=G\left(\frac{2 \mu-\lambda}{2 \mathrm{c}}\right)$. The above expression requires $d<x_{0}$ and $\lambda \leq 2 \mu$.Therefore, we need to consider two cases: $\lambda<2 \mu$ and $\lambda \geq 2 \mu$.

First we suppose $x_{1}>d>x_{0}$ under the case of $\mu<\lambda<2 \mu$.

Since $a(x)$ is an increasing function, we know $a(x)=1$ on $\left[m, x_{1}\right)$, which implies that the first equation of (4.1.5) becomes

$\frac{1}{2} \sigma^{2} a^{2} f^{\prime \prime}(x)+[a \lambda-(\lambda-\mu)] f^{\prime}(x)-\delta f(x)=0, \quad x \in\left[m, x_{1}\right)$

Therefore

$f_{1}(x)=C_{1}\left(x_{1}\right) e^{b_{1}\left(x+d-x_{0}\right)}+C_{2}\left(x_{1}\right) e^{b_{2}\left(x+d-x_{0}\right)}, \quad x \in\left[m, x_{1}\right) ;$

$f_{2}(x)=\beta_{1}\left(x-x_{1}\right)+f_{1}\left(x_{1}\right), \quad x \geq x_{1}$

Due to the continuity of the function $f^{\prime}(x)$ and $f^{\prime \prime}(x)$ at point $x_{1}$, we can derive that

$f_{1}^{\prime}(x)=C_{1}\left(x_{1}\right) b_{1} e^{b_{1}\left(x+d-x_{0}\right)}+C_{2}\left(x_{1}\right) b_{2} e^{b_{2}\left(x+d-x_{0}\right)}=\beta_{1}$,

$f_{1}^{\prime \prime}(x)=C_{1}\left(x_{1}\right) b_{1}^{2} e^{b_{1}\left(x+d-x_{0}\right)}+C_{2}\left(x_{1}\right) b_{2}^{2} e^{b_{2}\left(x+d-x_{0}\right)}=0$,

$C_{1}\left(x_{1}\right)=\frac{\beta_{1} b_{2}}{\mathrm{e}^{b_{1}\left(x_{1}-x_{0}+d\right)} b_{1}\left(b_{2}-b_{1}\right)}, C_{2}\left(x_{1}\right)=\frac{\beta_{1} b_{1}}{\mathrm{e}^{b_{2}\left(x_{1}-x_{0}+d\right)} b_{2}\left(b_{1}-b_{2}\right)}$

i.e.

From $f(m)=0$, we have

$f_{1}(m)=C_{1}\left(x_{1}\right) e^{b_{1}\left(m+d-x_{0}\right)}+C_{2}\left(x_{1}\right) e^{b_{2}\left(m+d-x_{0}\right)}=0$,

which implies that $x_{1}$ is a solution of (3.1). Using Lemma 3.1, we have $x_{1}=x_{1_{*}}$. Similarly, if $x_{1}=x_{1_{*}}$, then $f(m)=0$. So $f(m)=0 \Leftrightarrow x_{1}=x_{1_{*}}$.

We will prove that $f$ satisfies (4.1.2)-(4.1.4). Noticing that $\beta_{1}<1, \beta_{2}>1$, it suffices to prove the following:

$\left\{\begin{array}{l}\max _{a \in[0,1]}\left\{\frac{1}{2} \sigma^{2} a^{2} f^{\prime \prime}(x)+[a \lambda-(\lambda-\mu)] f^{\prime}(x)-\delta f(x)\right\} \leq 0, \\ f^{\prime}(x) \geq \beta_{1}, f^{\prime}(x) \leq \beta_{2}, m \leq x<x_{1_{*}} ; \\ \max _{a \in[0,1]}\left\{\frac{1}{2} \sigma^{2} a^{2} f^{\prime \prime}(x)+[a \lambda-(\lambda-\mu)] f^{\prime}(x)-\delta f(x)\right\} \leq 0, \\ x \geq x_{1_{*}} .\end{array}\right.$

The proof is as follows: for $x \geq x_{1_{*}}$,

$f_{2}(x)=\beta_{1}\left(x-x_{1_{*}}\right)+f_{1}\left(x_{1_{*}}\right)$, we have $\mu \beta_{1}-\delta f_{1}\left(x_{1_{*}}\right)=0$.

Therefore

$$
\begin{aligned}
& \max _{a \in[0,1]}\left\{\frac{1}{2} \sigma^{2} a^{2} f_{2}{ }^{\prime \prime}(x)+[a \lambda-(\lambda-\mu)] f_{2}{ }^{\prime}(x)-\delta f_{2}(x)\right\} \\
= & \max _{a \in[0,1]}\left\{[a \lambda-(\lambda-\mu)] f_{2}{ }^{\prime}(x)-\delta f_{2}(x)\right\} \\
= & \mu \beta_{1}-\delta f_{1}\left(x_{1_{*}}\right)-\delta \beta_{1}\left(x-x_{1_{*}}\right) \leq 0
\end{aligned}
$$

Using the same way as in Højgaard and Taksar [2], it is easy to prove that

$$
\max _{a \in[0,1]}\left\{\frac{1}{2} \sigma^{2} a^{2} f_{1}^{\prime \prime}(x)+[a \lambda-(\lambda-\mu)] f_{1}^{\prime}(x)-\delta f_{1}(x)\right\} \leq 0
$$

holds for $m \leq x<x_{1_{*}}$.

Since $f_{1}^{\prime}(x)=\frac{\beta_{1} b_{1} b_{2}}{b_{2}-b_{1}}\left[e^{b_{1}\left(m-x_{k_{k}}\right)}-e^{b_{2}\left(m-x_{l_{k}}\right)}\right] \leq 0$,

then $f^{\prime}(x)$ is a decreasing function on $\left[m, x_{1_{*}}\right]$. Moreover, $f^{\prime}\left(x_{1_{*}}\right)=\beta_{1}$ and $f^{\prime}(x) \geq \beta_{1}$ are obvious.

The problem remaining is to prove that the solution $f$ satisfies $\Im f(x) \leq f(x): f(y)+\beta_{1}(x-y)-K-f(x) \Leftrightarrow$

$\int_{y}^{x}\left(\beta_{1}-f^{\prime}(\partial)\right) d \partial-K \leq \int_{m}^{x}\left(\beta_{1}-f^{\prime}(\partial)\right) d \partial-K \leq 0$. The proof of (4.1.4) is as follows.

Since $f^{\prime}(m)=\frac{\beta_{1} b_{2}}{b_{2}-b_{1}} e^{b_{1}\left(m-x_{1_{*}}\right)}+\frac{\beta_{1} b_{1}}{b_{1}-b_{2}} e^{b_{2}\left(m-x_{1_{0}}\right)}=h\left(x_{1, *}\right), x_{1,} \leq x_{1_{. .}} \quad$ and $h(x)$ is a strictly increasing function, we have $f^{\prime}(m)=h\left(x_{1_{*}}\right) \leq h\left(x_{1_{* *}}\right)=\beta_{2}$ by Lemma 3.2.

\subsection{The solution to the problem with equity issuance}

In this subsection, our aim is to maximize the expected discounted dividends payout minus the expected discounted equity issuance over all reinsurance, dividends payout and equity issuance strategies. This kind of insurance companies will never go bankrupt.

Theorem 4.2. Assume that $x_{1_{*}} \geq x_{1_{* *}}$, where $x_{1_{*}}$ and $x_{1_{* *}}$ are defined in Lemmas 3.1 and 3.2. Then the function $g$ defined by

$$
g(x)=\left\{\begin{aligned}
g_{1}(x)= & C_{1}\left(x_{1_{s *}}\right) e^{b_{1}\left(x+d-x_{0}\right)}+C_{2}\left(x_{1_{* *}}\right) e^{b_{2}\left(x+d-x_{0}\right)}, \\
& m \leq x<x_{1_{s *}}, \\
g_{2}(x)= & \beta_{1}\left(x-x_{1_{* *}}\right)+g_{1}\left(x_{1_{* *}}\right), \\
& x \geq x_{1_{s s}},
\end{aligned}\right.
$$

satisfies the following HJB equation and the boundary conditions:

$$
\max \left\{\max _{a \in[0,1]}\left\{\frac{1}{2} \sigma^{2} a^{2} g^{\prime \prime}(x)+[a \lambda-(\lambda-\mu)] g^{\prime}(x)-\delta g(x)\right\}, \beta_{1}-f^{\prime}(x),\right.
$$

$\left.\mathfrak{J} g(x)-g(x), g^{\prime}(x)-\beta_{2}\right\}=0$,

$g^{\prime}(m) \geq 0$

where $b_{1}, b_{2}$ are the same as in Lemma 3.1, $C_{1}\left(x_{1 。}\right), C_{1}\left(x_{1 . .}\right)$ are defined as same as in Theorem 4.1 by replacing $x_{1_{*}}$ with $x_{1_{* *}}$.

Proof. Considering the time value of money leads us to the conclusion that it is optimal to postpone the new equity issuance as long as possible. If we issue equity at the reserve $n$ prior to $m, g^{\prime}(n)=\beta_{2}$ and $g^{\prime}(x)$ is a decreasing function, so $g^{\prime \prime}(n)$ must be 0 to meet the requirement $g^{\prime}(x) \leq \beta_{2}$. But it is not compatible with $a \in[0,1]$. Thus we know that it is optimal to issue equity only when the reserves become $m$.

\section{Volume 5 Issue 6, June 2016 www.ijsr.net}




\section{International Journal of Science and Research (IJSR) \\ ISSN (Online): 2319-7064}

Index Copernicus Value (2013): 6.14 | Impact Factor (2015): 6.391

By the same argument as in Theorem 4.1, we know the function $g$ should be characterized by

$$
\begin{aligned}
& g^{\prime}(m)=\beta_{2} \\
& \left\{\begin{array}{l}
\max _{a \in[0,1]}\left\{\frac{1}{2} \sigma^{2} a^{2} g^{\prime \prime}(x)+[a \lambda-(\lambda-\mu)] g^{\prime}(x)-\delta g(x)\right\}=0, \\
m \leq x<x_{1}, \\
g^{\prime}(x)=\beta_{1}, \quad x \geq x_{1}, \\
g^{\prime \prime}(x)=0, \quad x \geq x_{1} .
\end{array}\right.
\end{aligned}
$$

Doing the same procedures as in proof of Theorem 4.1, we can prove the function $g(x)$ of (4.2.4) and (4.2.5) has the same form as $f(x)$, and $x_{1}$ satisfies the following equation

$\frac{\beta_{1} b_{2}}{b_{2}-b_{1}} e^{b_{1}\left(m-x_{1}\right)}+\frac{\beta_{1} b_{1}}{b_{1}-b_{2}} e^{b_{2}\left(m-x_{1}\right)}=\beta_{2}$.

By Lemma 3.2, we have $x_{1}=x_{1_{* * *}}$ and $x_{1_{* * *}}>m$.

We will prove that the solution $g$ satisfies the conditions mentioned in Theorem 4.2. It suffices to prove the following: $\left\{\begin{array}{l}\max _{a \in[0,1]}\left\{\frac{1}{2} \sigma^{2} a^{2} g^{\prime \prime}(x)+[a \lambda-(\lambda-\mu)] g^{\prime}(x)-\delta g(x)\right\} \leq 0, \\ g^{\prime}(x) \geq \beta_{1}, g^{\prime}(x) \leq \beta_{2}, m \leq x<x_{1, \mu} ; \\ \max _{a \in[0,1]}\left\{\frac{1}{2} \sigma^{2} a^{2} g^{\prime \prime}(x)+[a \lambda-(\lambda-\mu)] g^{\prime}(x)-\delta g(x)\right\} \leq 0, \\ x \geq x_{1, \ldots},\end{array}\right.$

and $\mathfrak{I} g(x) \leq g(x)$.

Using the similar procedures as in Section 4.1, we can prove the above affirms.

We will verify $g(m) \geq 0$, i.e.

$$
\begin{aligned}
g(m) & =\frac{\beta_{1} b_{2}}{b_{1}\left(b_{2}-b_{1}\right)} e^{b_{1}\left(m-x_{1+*}\right)}+\frac{\beta_{1} b_{1}}{b_{2}\left(b_{1}-b_{2}\right)} e^{b_{2}\left(m-x_{1+*}\right)} \\
& =k\left(x_{1_{* *}}\right) \geq k\left(x_{1_{*}}\right) \\
& =\frac{\beta_{1} b_{2}}{b_{1}\left(b_{2}-b_{1}\right)} e^{b_{1}\left(m-x_{1 *}\right)}+\frac{\beta_{1} b_{1}}{b_{2}\left(b_{1}-b_{2}\right)} e^{b_{2}\left(m-x_{1 *}\right)} .
\end{aligned}
$$

(ii) $0 \leq d<x_{0}$

By the same argument as in (i), we can get the Lemmas and Theorems that are similar to Lemma 3.1, Lemma 3.2 and Theorem 4.1, Theorem 4.2, where $\bar{f}(x)$ and $\bar{g}(x)$ are defined as follow. The corresponding value functions are defined by $V_{A_{1}}(x)$ and $V_{B_{1}}(x)$, respectively.

$$
\bar{f}(x)=\left\{\begin{array}{c}
\bar{f}_{1}(x)=\left(C_{1}\left(x_{1^{*}}\right)+C_{2}\left(x_{1^{*}}\right)\right) e^{-\int_{x}^{x_{0}-d} \frac{1}{\mathrm{G}^{-1}(y+d)} d y}, \\
m \leq x<x_{0}-d, \\
\bar{f}_{2}(x)=C_{1}\left(x_{1^{*}}\right) e^{b_{1}\left(x+d-x_{0}\right)}+C_{2}\left(x_{1^{*}}\right) e^{b_{2}\left(x+d-x_{0}\right)}, \\
x_{0}-d \leq x<x_{1^{*}}, \\
\bar{f}_{3}(x)=\beta_{1}\left(x-x_{1^{*}}\right)+\bar{f}_{2}\left(x_{1^{*}}\right), \\
x \geq x_{1^{*}},
\end{array}\right.
$$

where

$$
C_{1}\left(x_{1^{*}}\right)=\frac{\beta_{1} b_{2}}{\mathrm{e}^{b_{1}\left(x_{1^{*}}-x_{0}+d\right)} b_{1}\left(b_{2}-b_{1}\right)}, C_{2}\left(x_{1^{*}}\right)=\frac{\beta_{1} b_{1}}{\mathrm{e}^{b_{2}\left(x_{1^{*}}-x_{0}+d\right)} b_{2}\left(b_{1}-b_{2}\right)} ;
$$

$$
\bar{g}(x)=\left\{\begin{array}{c}
\bar{g}_{1}(x)=\left(C_{1}\left(x_{1^{* *}}\right)+C_{2}\left(x_{1^{*}}\right)\right) e^{-\int_{x}^{x_{0}-d} \frac{1}{\mathrm{G}^{-1}(y+d)} d y}, \\
m \leq x<x_{0}-d, \\
\bar{g}_{2}(x)=C_{1}\left(x_{1^{* *}}\right) e^{b_{1}\left(x+d-x_{0}\right)}+C_{2}\left(x_{1^{* *}}\right) e^{b_{2}\left(x+d-x_{0}\right)}, \\
x_{0}-d \leq x<x_{1^{* *}}, \\
\bar{g}_{3}(x)=\beta_{1}\left(x-x_{1^{* *}}\right)+\bar{g}_{2}\left(x_{1^{* *}}\right), \\
x \geq x_{1^{* *}},
\end{array}\right.
$$

where

$$
C_{1}\left(x_{1^{*}}\right)=\frac{\beta_{1} b_{2}}{\mathrm{e}^{b_{1}\left(x_{1^{* m}}-x_{0}+d\right)} b_{1}\left(b_{2}-b_{1}\right)}, C_{2}\left(x_{1^{*}}\right)=\frac{\beta_{1} b_{1}}{\mathrm{e}^{b_{2}\left(x_{1^{* m}}-x_{0}+d\right)} b_{2}\left(b_{1}-b_{2}\right)} .
$$

(II) $\lambda \leq 2 \mu$.

In this case, the company does not need to reinsure, i.e. $a(x) \equiv 1$ for all $x \geq m$. By the same argument as in (i), we can get the Lemmas and Theorems that are similar to Lemma 3.1, Lemma 3.2 and Theorem 4.1, Theorem 4.2, where $\tilde{f}(x)$ and $\tilde{g}(x)$ are defined as follow. The corresponding value functions are defined by $V_{A_{2}}(x)$ and $V_{B_{2}}(x)$, respectively.

$\tilde{f}(x)= \begin{cases}\tilde{f}_{1}(x)=C_{11}\left(x_{1_{*}}\right) e^{b_{1}(x+d)}+C_{21}\left(x_{1 *}\right) e^{b_{2}(x+d)}, & m \leq x<x_{1 *} ; \\ \tilde{f}_{2}(x)=\beta_{1}\left(x-x_{1_{*}}\right)+\tilde{f}_{1}\left(x_{1_{*}}\right), & x \geq x_{1_{*}},\end{cases}$

where

$$
C_{11}\left(x_{1,}\right)=\frac{\beta_{1} b_{2}}{\mathrm{e}^{b_{1}\left(x_{10}+d\right)} b_{1}\left(b_{2}-b_{1}\right)}, C_{21}\left(x_{1 \mathrm{o}}\right)=\frac{\beta_{1} b_{1}}{\mathrm{e}^{b_{2}\left(x_{10}+d\right)} b_{2}\left(b_{1}-b_{2}\right)}
$$

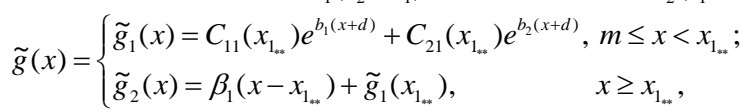

where

$C_{11}\left(x_{1 \ldots}\right)=\frac{\beta_{1} b_{2}}{\mathrm{e}^{b_{1}\left(x_{1+\infty}+d\right)} b_{1}\left(b_{2}-b_{1}\right)}, C_{21}\left(x_{1 \ldots}\right)=\frac{\beta_{1} b_{1}}{\mathrm{e}^{b_{2}\left(x_{1 .+口}+d\right)} b_{2}\left(b_{1}-b_{2}\right)}$.

\section{The Solution to the General Problem}

We now study the optimal control problem without any restriction on the issuance of equity.

Theorem 5.1. Let concave function $\Gamma(x) \in C^{2}$ satisfy the following HJB equation and boundary condition : for $x \geq m$, $\max \left\{\max _{a \in[0,1]}\left\{\frac{1}{2} \sigma^{2} a^{2} \Gamma^{\prime \prime}(x)+[a \lambda-(\lambda-\mu)] \Gamma^{\prime}(x)-\delta \Gamma(x)\right\}, \beta_{1}-\Gamma^{\prime}(x)\right.$,

$\left.\mathfrak{I} \Gamma(x)-\Gamma(x), \Gamma^{\prime}(x)-\beta_{2}\right\}=0$,

$\max \left\{-\Gamma(m), \Gamma^{\prime}(m)-\beta_{2}\right\}=0$.

Then $\Gamma(x) \geq V(x, \pi)$ for any admissible policy $\pi$.

Proof. Since $\Gamma(x)$ is a concave, increasing and continuous function on $[m, \infty)$. From $\Im \Gamma(x) \leq \Gamma(x)$, we know $\Gamma(x-\xi)-\Gamma(x) \leq K-\beta_{1} \xi, \forall x \geq m, \xi>0$.

For a policy $\pi \in \Pi$, we note $\Theta=\left\{s: L_{s}^{\pi} \neq L_{s^{-}}^{\pi}\right\}=\left\{\tau_{1}^{\pi}, \tau_{2}^{\pi}, \ldots \ldots.\right\}$ and $\Theta^{\prime}=\left\{s: G_{s}^{\pi} \neq G_{s^{-}}^{\pi}\right\}$. $G_{t}^{\pi, c}=G_{t}^{\pi}-\sum_{s \in \Theta^{\prime}, s \leq t}\left(\mathrm{G}_{\mathrm{s}}^{\pi}-\mathrm{G}_{\mathrm{s}^{-}}^{\pi}\right)$ is the continuous part of $G_{t}^{\pi}$. By Itô formula,

$$
\begin{aligned}
e^{-\delta\left(t \wedge \tau^{\pi}\right)} \Gamma\left(\mathrm{R}_{t \wedge \tau^{\pi}}^{\pi}\right)= & \Gamma(x)+\int_{0}^{t \wedge \tau^{\pi}} e^{-\delta s} \vartheta \Gamma\left(\mathrm{R}_{\mathrm{s}}^{\pi}\right) d s \\
& +\int_{0}^{t \wedge \tau^{\pi}} e^{-\delta \delta} \sigma a_{s}^{\pi} \Gamma^{\prime}\left(\mathrm{R}_{\mathrm{s}}^{\pi}\right) d B s \\
& +\int_{0}^{t \wedge \tau^{\pi}} e^{-\delta \delta} \Gamma^{\prime}\left(\mathrm{R}_{\mathrm{s}}^{\pi}\right) d G_{s}^{\pi, c} \\
& +\sum_{s \in \Theta \cup \Theta^{\prime}, s \leq t \wedge \tau^{\pi}} e^{-\delta \delta}\left[\Gamma\left(\mathrm{R}_{\mathrm{s}}^{\pi}\right)-\Gamma\left(\mathrm{R}_{\mathrm{s}^{-}}^{\pi}\right)\right],
\end{aligned}
$$




\section{International Journal of Science and Research (IJSR) \\ ISSN (Online): 2319-7064}

Index Copernicus Value (2013): 6.14 | Impact Factor (2015): 6.391

where $\vartheta \Gamma(x)=\frac{1}{2} \sigma^{2} a^{2} \Gamma^{\prime \prime}(x)+[a \lambda-(\lambda-\mu)] \Gamma^{\prime}(x)-\delta \Gamma(x)$,

$$
\begin{aligned}
\sum_{s \in \Theta \cup \Theta^{\prime}, s \leq t \wedge \tau^{\pi}} e^{-\delta s}\left[\Gamma\left(\mathrm{R}_{\mathrm{s}}^{\pi}\right)-\Gamma\left(\mathrm{R}_{\mathrm{s}^{-}}^{\pi}\right)\right]= & \sum_{s \in \Theta, s \leq t \wedge \tau^{\pi}} e^{-\delta s}\left[\Gamma\left(\mathrm{R}_{\mathrm{s}}^{\pi}\right)-\Gamma\left(\mathrm{R}_{\mathrm{s}^{-}}^{\pi}\right)\right] \\
& +\sum_{s \in \Theta^{\prime}, s \leq t \wedge \tau^{\pi}} e^{-\delta s}\left[\Gamma\left(\mathrm{R}_{\mathrm{s}}^{\pi}\right)-\Gamma\left(\mathrm{R}_{\mathrm{s}^{-}}^{\pi}\right)\right] \\
& \leq \sum_{s \in \Theta^{\prime}, s \leq t \wedge \tau^{\pi}} e^{-\delta \delta} \beta_{2}\left(\mathrm{G}_{\mathrm{s}}^{\pi}-\mathrm{G}_{\mathrm{s}^{-}}^{\pi}\right) \\
& -\sum_{i=1}^{\infty} e^{-\delta \tau_{i}^{\pi}}\left(-\mathrm{K}+\beta_{1} \xi_{i}^{\pi}\right) I_{\left\langle\tau_{i}^{\pi} \leq t \wedge \tau^{\pi}\right|} .
\end{aligned}
$$

By substituting this inequality into the above equation and taking expectation on both sides, we obtain

$$
\begin{aligned}
E\left\{e^{-\delta\left(t \wedge \tau^{\pi}\right)} \Gamma\left(\mathrm{R}_{t \wedge \tau^{\pi}}^{\pi}\right)\right\} \leq & \Gamma(x)+E\left\{\int_{0}^{t \wedge \tau^{\pi}} e^{-\delta \delta} \beta_{2} d G_{s}^{\pi}\right\} \\
& -E\left\{\sum_{i=1}^{\infty} e^{-\delta \tau_{i}^{\pi}}\left(-\mathrm{K}+\beta_{1} \xi_{i}^{\pi}\right) I_{\left\{\tau_{i}^{\pi} \leq t \wedge \tau^{\pi}\right\}}\right\} .
\end{aligned}
$$

By the definition of $\tau^{\pi}$ and $\beta_{1} \leq \Gamma^{\prime}(x) \leq \beta_{2}$, it is

easy to prove that

$\liminf e^{-\delta\left(t \wedge \tau^{\pi}\right)} \Gamma\left(\mathrm{R}_{t \wedge \tau^{\pi}}^{\pi}\right)+e^{-\delta \tau^{\pi}} \Gamma(m) I_{\left\{\tau^{\pi}<\infty\right\}}+\liminf e^{-\delta \delta} \Gamma\left(\mathrm{R}_{t}^{\pi}\right) I_{\left\{\tau^{\pi}=\infty\right\}} \geq 0$.

So we deduce that

$V(x, \pi)=E\left[\sum_{i=1}^{\infty} e^{-\delta \tau_{i}^{\pi}}\left(-K+\beta_{1} \xi_{i}^{\pi}\right) I_{\left\{\tau_{i}^{\pi} \leq \tau^{\pi}\right\}}-\int_{0}^{\tau^{\pi}} \mathrm{e}^{-\delta s} \beta_{2} d G_{s}^{\pi}\right] \leq \Gamma(x)$.

The main results of this paper are the following.

Theorem 5.2. (I) $\mu<\lambda<2 \mu$.

(i) $x_{1}>d>x_{0}$.

$x_{1_{*}}, x_{1_{* *}}$ are given in Lemmas, $V(x), f(x)$ and $g(x)$ are

defined by (2.4), (4.1.1) and (4.2.1) respectively. $V_{A}(x)$ and $V_{B}(x)$ are defined in Section 4.

If $x_{1,} \leq x_{1_{w ⿻}}$, then $V(x)=f(x)=V_{A}(x)$. The optimal policy $\pi^{*}=\left(a^{\pi^{*}}, L^{\pi^{*}}, G^{\pi^{*}}\right)$

satisfies the following

$$
\left\{\begin{array}{l}
R_{t}^{\pi^{*}}=x+\int_{0}^{t}\left[a_{s}^{\pi^{*}} \lambda-(\lambda-\mu)\right] d_{s}+\int_{0}^{t} a_{s}^{\pi^{*}} d B_{s}-\sum_{n=1}^{\infty} I_{\left\{\tau_{i}^{*^{*}} \leq t\right.} \xi_{i}^{\pi^{*}}, \\
R_{t}^{\pi^{*} \leq x_{1_{*}},} \\
\int_{0}^{\infty} I_{\left\{t: R_{t}^{\pi^{*}}<x_{1_{1}}\right\}}(t) d L_{t}^{\pi^{*}}=0, \\
G_{t}^{\pi^{*}}=0,
\end{array}\right.
$$

where $a_{t}^{\pi^{*}}=a\left(R_{t}^{\pi^{*}}\right)$.

If $x_{1_{*}} \geq x_{1_{* *}}$, then $V(x)=f(x)=V_{B}(x)$. The optimal

policy $\pi^{* *}=\left(a^{\pi^{* *}}, L^{\pi^{* *}}, G^{\pi^{* *}}\right)$ satisfies the following

$$
\left\{\begin{array}{l}
R_{t}^{\pi^{* *}}=x+\int_{0}^{t}\left[a \pi^{* *} \lambda-(\lambda-\mu) d_{s}+\int_{0}^{t} a_{s}^{\pi^{* *}} d B_{s}-\sum_{n=1}^{\infty} I_{\left\{\tau_{i}^{\pi^{* *}} \leq t\right\}} \xi_{i}^{\pi^{* *}}+G_{t}^{\pi^{* *}},\right. \\
m \leq R_{t}^{\pi^{* *}} \leq x_{1 . .}, \\
\int_{0}^{\infty} I_{\left\{t: R_{t}^{\pi^{* *}}<x_{1.0}\right\}}(t) d L_{t}^{\pi^{* *}=0,} \\
\int_{0}^{\infty} I_{\left\{t: R_{t}^{\left.\pi^{* *}<m\right\}}\right.}(t) d G_{t}^{\pi^{* *}}=0,
\end{array}\right.
$$

where $a_{t}^{\pi^{* *}}=a\left(R_{t}^{\pi^{* *}}\right)$.

According to Lions and Sznitman [14] we know that the processes $\pi^{*}=\left(a^{\pi^{*}}, L^{\pi^{*}}, G^{\pi^{*}}\right)$ and $\pi^{* *}=\left(a^{\pi^{* *}}, L^{\pi^{* *}}, G^{\pi^{* *}}\right)$ are uniquely determined by (5.4) and (5.5).

(ii) $0 \leq d<x_{0}$.

All the results are similar to $x_{1}>d>x_{0}$.

(II) $\lambda \leq 2 \mu$.
All the results are same to the case $x_{1}>d>x_{0}$. In this case, the insurance company doesn't need to reinsure.

Proof. (I) If $x_{1_{*}} \leq x_{1_{* *}}$, the function $f(x)$ satisfies the HJB equation and boundary conditions. And $f(x)$ also satisfies conditions (5.1) and (5.2) in Theorem 5.1. So $f(x) \geq V(x) \geq V_{A}(x)$ by Theorem 5.1. We will prove $f(x)=V(x)$ corresponding to $\pi^{*}$. Applying generalized Itô formula, we obtain $\vartheta f\left(\mathrm{R}_{t \wedge \tau^{\pi^{*}}}^{\pi^{*}}\right)=0$ and

$$
\begin{aligned}
e^{-\delta\left(t \wedge \tau^{\pi^{*}}\right)} f\left(\mathrm{R}_{t \wedge \tau^{\pi^{*}}}^{\pi^{*}}\right)= & f(x)+\sigma \int_{0}^{t \wedge \pi^{\pi^{*}}} e^{-\delta s} a\left(\mathrm{R}_{t}^{\pi^{*}}\right) f^{\prime}\left(\mathrm{R}_{s}^{\pi^{*}}\right) d B_{s} \\
& -\sum_{i=1}^{\infty} e^{-\delta \tau_{i}^{\pi^{*}}}\left(-\mathrm{K}+\beta_{1} \xi_{i}^{\pi^{*}}\right) I_{\left\{\tau_{i}^{\pi^{*}} \leq t \wedge \tau^{\pi^{*}}\right\}} .
\end{aligned}
$$

where $\tau^{\pi^{*}}=\inf \left\{t \geq 0: R_{t}^{\pi^{*}}<m\right\}$. Because

$\liminf e^{-\delta\left(t \wedge \tau^{\pi^{*}}\right)} f\left(\mathrm{R}_{t \wedge \tau^{\pi^{*}}}^{\pi^{*}}\right)=e^{-\delta \tau^{\pi^{*}}} f(m)=0$, taking expectation

at both sides of (5.6), we get

$$
f(x)=E\left[\sum_{i=1}^{\infty} e^{-\delta \tau_{i}^{\pi^{*}}}\left(-K+\beta_{1} \xi_{i}^{\pi^{*}}\right) I_{\left\{\tau_{i}^{\pi^{*}} \leq \tau^{\pi^{*}}\right\}}\right]=V\left(x, \pi^{*}\right) .
$$

So $f(x)$ is the value function corresponding to $\pi^{*}$, and $f(x) \leq V_{A}(x)$. Using the results $f(x) \geq V(x) \geq V_{A}(x)$, we have $f(x)=V(x)=V_{A}(x)$.

If $x_{1_{*}} \geq x_{1_{* *}}, g(x)$ defined in (4.2.1) satisfies the HJB equation and boundary conditions. Thus $g(x)$ satisfies conditions (5.1) and (5.2) in Theorem 5.1. So $g(x) \geq V(x) \geq V_{B}(x)$ by Theorem 5.1. We will prove $g(x)=V(x)$ corresponding to $\pi^{* *}$. Applying generalized Itô formula, we obtain $\vartheta f\left(\mathrm{R}_{t \wedge \tau^{\pi^{*}}}^{\pi^{* *}}\right)=0$ and

$$
\begin{aligned}
& e^{-\delta\left(t \wedge \tau^{\pi^{* *}}\right)} g\left(\mathrm{R}_{t \wedge \tau^{\pi^{* *}}}^{\pi^{* *}}\right)=g(x)+\int_{0}^{t \wedge \tau^{\pi^{* *}}} e^{-\delta s} \beta_{2} d G_{s}^{\pi^{* \prime}} \\
& +\sigma \int_{0}^{t \wedge \tau^{\pi^{* \prime}}} e^{-\delta s} a\left(\mathbf{R}_{t}^{\pi^{* *}}\right) f^{\prime}\left(\mathbf{R}_{s}^{\pi^{* \prime}}\right) d B_{s} \\
& -\sum_{i=1}^{\infty} e^{-\delta \tau_{i}^{\pi^{* *}}}\left(-\mathrm{K}+\beta_{1} \xi_{i}^{\pi^{* *}}\right) I_{\left\{\tau_{i}^{\pi^{* *}} \leq t \wedge \tau^{\pi^{* *}}\right\}},
\end{aligned}
$$

where $\tau^{\pi^{* *}}=\inf \left\{t \geq 0: R_{t}^{\pi^{* *}}<m\right\}$. Since

$\liminf e^{-\delta\left(t \wedge \tau^{\pi^{* *}}\right)} g\left(\mathrm{R}_{t \wedge \tau^{\pi^{* \prime}}}^{\pi^{* *}}\right)=0$, by taking expectation

at both sides of (5.7), we get

$$
\begin{aligned}
g(x) & =E\left[\sum_{i=1}^{\infty} e^{-\delta \tau_{i}^{\pi^{* *}}}\left(-K+\beta_{1} \xi_{\mathrm{i}}^{\pi^{* *}}\right) I_{\left\{\tau_{i}^{\pi^{* *}} \leq \tau^{\pi^{* *}}\right\}}-\int_{0}^{\tau^{\pi^{* *}}} \mathrm{e}^{-\delta \delta} \beta_{2} d G_{s}^{\pi^{\pi^{* *}}}\right] \\
& =V\left(x, \pi^{* *}\right) .
\end{aligned}
$$

So $g(x)$ is the value function corresponding to $\pi^{* *}$, and $g(x) \leq V_{B}^{(5,5)}$. Using the results $g(x) \geq V(x) \geq V_{B}(x)$, we have $g(x)=V(x)=V_{B}(x)$. The proof of (ii) and (II) is similar to (i), so we omit it here.

\section{Conclusion}

In this paper, we consider the optimal dividend and financing control problem in the risk model with non-cheap proportional reinsurance. The management of the company controls the reinsurance rate, dividends payout and the equity issuance to maximize the expected present value of the dividends payout minus the equity issuance until the ruin time. To be more

\section{Volume 5 Issue 6, June 2016 www.ijsr.net}




\section{International Journal of Science and Research (IJSR) \\ ISSN (Online): 2319-7064}

Index Copernicus Value (2013): 6.14 | Impact Factor (2015): 6.391

realistic, we assume the minimal reserve restrictions and consider the fixed and proportional transaction costs. The former cost is generated by the advisory and consulting as well as the latter is generated by the tax. It is the first time to study non-cheap proportional reinsurance in an insurance model with this method to solve the optimal control problem, which construct two categories of suboptimal control problems, one is the classical model without equity issuance, the other never goes bankrupt by equity issuance. We verify the optimal strategy and the value function with the corresponding solution in either category of suboptimal models, tightly connecting with the relationships among the parameters.

\section{Acknowledgements}

We are very gratrful to the careful reading of the manuscript, correction of errors, valuable suggestions which improved this paper very much.

\section{References}

[1] De Finetti, B., "Su un'impostazione alternativa dell teoria collettiva del rischio," Transactions of the XVth International Congress of Actuaries, Vol.2, pp. 433-443, 1957.

[2] Højgaard, B., Taksar, M., "Controlling risk exposure and dividnds payout schemes: Insurance company example," Math. Finance, 9(2), pp. 153-182, 1999.

[3] Gerber, Hans U., Shiu, Elias S.W., “ Optimal dividends: analysis with Brownian motion,” N. Am. Actuar. J., 8(1), pp. 1-20, 2004.

[4] Cadenillas, A., Choulli, T., Taksar. M., Zhang, L., " Classical and impulse stochastic control for the optimization of the divideng ang risk policies of an insurance firm," Math. Finance, 16(1), pp.181-202, 2006.

[5] Avanzi, B., Gerber, H.U., Shiu, E.S.W., “Optimal dividends in the dual model," Insur. Math. Econ., 41(1), pp. 111-123, 2007.

[6] Sethi, S.P., Taksar, M., " Optimal financing of a corporation subject to random returns," Math. Finance, 12(2), pp.155-172, 2002.

[7] Løkka, A., Zervos, M., "Optimal dividend and issuance of equity policies in the presence of proportional costs," Insur. Math. Econ., 42(3), pp. 954-961, 2008.

[8] Asmussen, S., Højgaard, B., Taksar, M., "Optimal risk control and dividend distribution policies: example of excess-of-loss reinsurance for an insurance corporation," Finance Stochast., 4(3), pp. 299-324, 2000.

[9] Choulli, T., Taksar, M., Zhou, X.Y., "Excess-of-loss reinsurance for acompany with debt liability and constraints on risk reduction," Quant.Finance, 1, pp. 573-596, 2001.

[10] Centeno, M., " Dependent risks and excess of loss reinsurance," Insur.Math. Econ., 37(2), pp.229-238, 2005.

[11]He, L., Liang, Z.X., “ Optimal financing and dividend control of the insurance company with fixed and proportional transaction costs," Insur. Math. Econ., 42, pp. 976-983, 2008.
[12]He, L., Liang, Z.X., “ Optimal financing and dividend control of the insurance company with fixed and proportional transaction costs," Insur. Math. Econ., 44(1), pp. 88-94, 2009.

[13] Wendell, H., Fleming, H. Mete Soner., " Controlled Markov Processes and Viscosity Solutions," SpringerVerllag, New York, 1993.

[14]Lions, P.-L., Sznitman, A.S., “ Stochastic dierential equations with reflecting boundary conditions," Comm. Pur. Appl. Math., 37, pp.511-537, 1984.

\section{Author Profile}

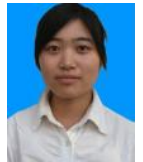

Ye Liu received the B.S. degree in Mathematics and Applied Mathematics from Handan College in 2014 , and now studying in the graduate school of Hebei university of technology, China.

Danfeng Zhao received the B.S. degree in Mathematics and Applied Mathematics from Xingtai College in 2014, and now studying in the graduate school of Hebei university of technology, China. 